\title{
Les gravures sur bambou, transcription authentique de la langue et de la pensée des Néo-Calédoniens
}

\author{
Marguerite et Georges Lobsiger-Dellenbach
}

Tous les peuples ont pensé et tous ont voulu matérialiser le signifié, concept de la réalité, par un signifiant, sa traduction phonétique ou graphique, quelquefois simultanée dans nombre de cas. Ce signifiant peut être mimique ou language. Sous sa forme graphique, il peut être pictographie, idéogramme, hiéroglyphe ou alphabet.

Les exemples ne manquent pas de sociétés éloignées du club raffiné des peuples-écrivains qui malgré leur isolement géographique et mental, ont inventé des procédés originaux d'expression graphique dont l'unique raison d'être est de représenter la parole. Au sens large du terme, ces écritures sont la mémoire et le conservatoire de cette parole. Dans son Cours de linguistique générale. F. de Saussure écrit: «...le mot écrit se mêle si intimement au mot parlé dont il est l'image qu'il finit par usurper le rôle principal...». On ne peut appliquer cette formule aux gravures sur bambous de Nouvelle-Calédonie, ces archives de la pensée canaque. Sous une forme tantôt figurative, tantôt abstraite, unissant simultanément l'image de la réalité au schéma et au symbole, suivant en cela la démarche intellectuelle du Néo-Calédonien, ces gravures dévoilent à leurs très rares déchiffeurs une réalité oubliée de nos jours par les descendants des artistes qui les incisèrent et de ceux qui écoutèrent les mainteneurs de traditions développer oralement les allusions inscrites sur ses aides-mémoire. L' "écriture» néo-calédonienne n'a jamais remplacé l'éloquence emphatique de mise lors des pilou, ces grandes fêtes claniques ou les strophes ardentes des poëmes épiques, comme le Chant de guerre des Méa ou encore les récits lyriques, comme le conte des Sœurs de Moaxa. Cette littérature orale fut récoltée au début de notre siècle par Maurice Leenhardt, qui sut les traduire non seulement mot à mot, mais encore leur donner une forme littéraire qui permettrait de les introduire dans quelque anthologie de la poésie orale du monde primitif.

Depuis trente ans, nous avons analysé et décrit assez de bambous gravés appartenant aux musées de Suisse et des autres pays d'Europe pour ne pas reconnaître dans ces humbles tracés l'expression parfaite de la langue et de la pensée de la région houaïlou, au centre de l'île. Cette gravure suit l'expression parlée avec une telle fidélité que, contrairement à l'opinion de F. de Saussure, qu'on peut ne pas suivre ici, le graphisme n'est pas un travestisse- ment de la parole, car il conserve avec modestie son rôle de représentation du signifiant phonétique. La gravure sur bambou explique, elle raconte, elle suggère surtout; elle sert de support à des allusions d'un récit plus complet, elle est un guide pour le récitant. Elle n'est pas une forme autonome d'expression, elle met en action la mémoire du lecteurrécitant, qui, en l'occurence, associe l'expression graphique et phonétique.

De là procède cette tournure elliptique et allusive des textes - on peut user ici de ce terme - gravés sur ces bambous de formats variables, avec des techniques allant de la médiocrité à la perfection, de la note cursive quasi sténographiée et hyperschématisée au tableautin de genre fignolé devant la case de travail par un artiste sensible aux encouragements et aux conseils judicieux des connaisseurs l'entourant au cours de sa gravure avec une dent de rat ou un fragment de carapace de crustacé, son seul burin.

Pour déchiffrer ces gravures souvent secrètes, on ne peut appliquer les méthodes statistiques, si à la mode ces temps. Ce procédé est utile pour déterminer quelques grandes lignes d'un ensemble mais inopérant pour l'étude de détail. Au contraire, il faut se pénétrer de l'esprit canaque, non par des procédés rationalistes, mais par une communion d'esprit avec ces "Sauvages» au facies brutal, à la réputation mérités de cannibales, qui, malgré ces tares rédhibitoires, ont toujours eu une étonnante faculté d'abstraction, faculté relevée par leurs meilleurs connaisseurs, Maurice Leenhardt, déjà nommé, et Jean Guiart, son compétent successeur. Le haut degré d'évolution de la pensée de ces anthropophages et de réflexion sur elle-même a frappé les observateurs de la vie psychique de ces paysans insulaires, de ces hommes d'un Néolithique vivace jusqu'à nos jours. La vie mythique accompagnait sans cesse le Canaque, rappelle $M$. Leenhardt, et elle lui dictait sa conduite et son comportement devant la nature, les dieux et les hommes.

$\mathrm{Au}$ début du 20ème siècle encore, ce mythe était toujours vivant. Ce n'était pas un simple fait ethnographiable ou un sujet d'enquêtes académiques. C'était une réalité profonde, car pour le Canaque et tous les peuples situés à son niveau, la réalité ne siège pas dans l'aspect fugace d'une apparence extérieure, mais dans la formule intérieure et éternelle, souvent invisible, celle qui fut créée par les An- 
cêtres surnaturels aux époques intemporelles. Vers 1900 , il n'y avait pas encore de hiatus entre la pensée du jour et celle d'hier, pas encore d'évolution destructrice ayant vidé le mythe de sa fonction régulatrice et protectrices. Aucune sécularisation ne l'avait transformé en allégorie puérile. Pour ces raisons, la lecture d'un bambou gravé exige une somme considérable de respect. La communion avec cette pensée mythique demande du déchiffreur un grand effort d'adaptation, au prix d'un véritable auto-lavage de cerveau, pour adapter ici une méthode fort blâmable quand elle est imposée du dehors. Pour penser et réagir comme un Canaque il doit assimiler les documents ethnologiques, il doit se pénétrer de toute la littérature recueillie, contes et légendes, dans le texte original, et dans les traductions mot à mot. Il doit connaître le vocabulaire et les synonymes ainsi que les canaquismes intraduisibles sans commentaires. Ce sont justement ces idiotismes typiques créant un fossé entre les deux mentalités qui sont les plus utiles lors d'un déchiffrement: grâce à ces décalages mentaux, il est permis de comprendre les bizarreries de sens de ces tableaux qui souvent, avec une maladresse émouvante et insistante, veulent suivre à tout prix l'expression phonétique. Le décrypteur peut parfois atteindre les premiers degrés de l'initiation à cette pensée éloignée de nos itinéraires mentaux. L'habitude, l'expérience et surtout la chance, le conduisent vers la compréhension de ces gravures où presque toujours la trame est constituée par les gestes agricoles relatifs à l'igname et au taro. Ils déterminent le calendrier sacré et profane, ainsi que le comportement individuel et collectif des habitants du village, comme l'imposition des tabous et leur levée saisonnières, les fêtes et les périodes d'austérité, dans le milieu arborisé par ces cannibales, qui, architectes-paysagistes d'instinct, surent toujours embellir leurs villages par le soin donné au cadre naturel et artificiel de leur habitat. Leur pensée non-cartésienne exige pour les comprendre un esprit libéré provisoirement du poids de nos catégories mentales: nos classifications sont inopérantes dans ce milieu cohérent axé sur d'autres coordonnées psychiques que les nôtres.

Les motifs géométriques, si abondants, permettent parfois au lecteur de mieux traduire les sentiments profonds des Canaques que leurs dessins réalistes, qui ne répondent pas, dans leur esprit, à la figuration exacte des choses et des êtres, mais toujours à des images phonétiques traduisant les concepts de la réalité, qu'elle soit naturelle ou mystique. Par exemple, sur un bambou de Genève deux registres de décorations apparentes nous ont permis de définir exactement le thème d'une frise humaine et de découvrir, grâce à leurs déterminatifs précis, qu'il s'agissait de sages dits "frappeurs de Soleil» chargés de provoquer la pluie. Leurs attributs, trop schématisées, n'avaient pu nous mettre sur la voie. Ici, le symbole a été plus clair que le schéma trop abscons. Un autre exemple apparait sur un bambou des collections du Musée des arts africains et océaniques, à Paris, encore non publié, qui nous paraît traduire quelques strophes de ce Chant de l'Eau déjà mentionné. Par son ambivalence, il serait incompréhensible à celui qui n'aurait pris plaisir à la lecture de ce poème remarquable avant de tenter le déchiffrement du bambou gravé.

Le support des gravures est donc un bambou, dont la hauteur dépasse très rarement deux mètres et dont le diamètre va de 20 à $70 \mathrm{~mm}$. Le trait n'est jamais arrondi et l'angle triomphe. Pour cette raison, ce que l'ethnographe décrit sous la forme d'une demi-lune, un sillon d'igname par exemple, devient un chevron sous le burin du graveur. L'igname est la plante masculine et sèche, uniquement arrosée par la pluie, alors que le taro, plante féminine et humide, exige l'irrigation artificielle. $\mathrm{Si}$ quelques bambous présentent des scènes réalistes, presque photographiques de la culture de ces plantes-mères de l'île, leur omniprésence dans l'esprit canaque est telle qu'une allusion suffit à déclencher toute une suite d'images et de prières. De là la présence constante de sillons d'ignames, files et rangs de chevrons hachurés: il faut les distinguer de la présence tout autant constante de l'eau, figurée par des lignes vivrées, avec laquelle on pourrait confondre les chevrons non hachurés. Cette représentation de l'eau par une ligne vivrée ou ondulée est universelle et on la retrouve à toutes les époques sous toutes les latitudes.

La difficulté de traduire minutieusement chaque détail des gestes agricoles si importants pour cette population rurale, qui se livre à la pêche surtout dans les périodes de soudure et qui n'apprécie la chair humaine que par raffinement de gourmets, crée ce que nous proposons de nommer des homogrammes, soit des images identiques représentant des réalités différentes. Nous savons que les façons culturales différenciant l'igname et le taro naissent de leur arrosage, naturel pour l'igname, artificiel pour le taro. Le mot ja (pétrir avec les pieds et défoncer le sol inondé des tarodières) entre dans la composition du terme nejawa, qui, au début signifiait pétrir cette terre, planter et arracher les taros) et qui, avec le temps, a pris le sens de tarodière. Il y a là deux idées connexes liées à l'eau boueuse de ces plantations. Cette eau boueuse des tarodières s'exprime par le signe géométrique formé de petits triangles (la terre retournée au bâton à fouir) et la ligne vivrée figurant l'eau aussi bien que l'aqueduc.

La même association des signifiants "terre et eau» peut aussi représenter la terre mouillée et non plus l'eau boueuse. Cette terre mouillée est celle des sillons d'ignames arrosés exclusivement par la pluie. 
Les deux éléments de ce binôme étant la terre et l'eau, leur représentation est donc identique. Leur figuration sur un bambou est tantôt réaliste, tantôt symbolique. Le déchiffreur doit à ce moment tenir compte du contexte et surtout des vignettes traduisant une période de l'année, sous l'aspect d'une époque de tabous sexuels (l'homme est alors privé de ses attributs virils), ou de levée de ces mêmes interdits sexuels (le couple apparait dans des poses ne laissant aucun doute quant à la libération sexuelle temporaire). Or, le calendrier rural de l'igname diffère de celui du taro. La connaissance de ces décalages agricoles et culturels est alors d'un grand secours. Il arrive, mais rarement, que le signe de l'eau, cette ligne vivrée horizontale soit bien définie comme pluie grâce à un déterminatif assez compliqué: ce signe peut être relié à un losange (nuage) par une ligne vivrée verticale (pluie et non plus simplement eau). L'explication se précise: la plantation symbolisée par des décors géométriques ou schématiques est bien celle de l'igname. Cette bande vivrée verticale peut aussi, suivant les cas, correspondre au concept de neto (tonnerre) qui a un sens de fertilité pour l'orant mais qui peut être une malédiction invoquée contre un village adversaire, avec le sens d'inondation. La tonnerre, annonciateur des orages bénéfiques pour les uns, maléfiques pour les ennemis, prend l'aspect de l'éclair. L'homogramme tonnerre-éclair s'associe à celui de eaufertilité et à celui de eau-inondation à la suite de ces parallélismes mentaux et verbaux. Le lézard, protecteur des récoltes, suit, lui aussi un tracé en zig-zag. Le contexte peut seul départager les hypothèses.

De tels homogrammes apparaissent également dans le signe de cendres, qui symbolise le deuil, la coquetterie et les étrangers. Le mot de signifie «cendres, symbole de deuil» tandis que les produits utilisés par les belles canaques pour blondir artificiellement les cheveux, comme les cendres, la chaux de coquillages brûlés et depuis quelques années, l'eau oxygénée se traduisent par ja. Ce mot de se retrouve dans le terme koedede qui signifie l'état des veuves, au corps noirci à la poudre de bancoul (champignon local) et aux cheveux blanchis à la cendre. Les cheveux clairs des étrangers sont traduits par le même signe graphique: c'est le pointillé des cendres que l'on note sous les marmites dessinées dans les cours. Le graphisme cendre traduit donc des concepts différents réunis par l'idée de peinture corporelle au moyen de matières pulvérisées. Les frappeurs du soleil, mentionnés antérieurement, ont eux aussi, le corps piqueté de points signifiant leur peinture corporelle et liturgique; nous n'aurions jamais compris ce sens de couleur sans les motifs abstraits les entourant.

Dans ce genre de méthode de traduction graphique de la parole et de la pensée, le Canaque suit exacte- ment dans son dessin les traits spécifiques du langage. L'image est subordonnée au mot. Le Canaque a été capable de reproduire avec une étonnante minutie les apports de la civilisation française. Il s'est complu à graver un nombre très grand de voiliers français, au gréement compliqué, avec une précision telle, qu'un spécialiste de la navigation à voile a pu nous définir une douzaine de types de voiliers allant du sloop au trois-mâts classique, et même reconnaître un bateau d'une compagnie bordelaise par un détail caractéristique figuré sur la coque du navire de cette compagnie de navigation. Ces mêmes Canaques ont su noter le détail différenciant le sémaphore du télégraphe optique, pourtant si ressemblants extérieurement. Nul ne peut donc les accuser de manquer de sens d'observation. Le graveur sait aussi user du déterminatif précis pour affirmer son dessin. Il est si prisonnier de sa langue qu'il est victime du poids de ses expressions verbales. Par exemple, il a été séduit par le fusil, à piston ou à percussion, porté, épaulé ou placé dans le ratelier ou formé en faisceau. Il mentionne avec soin la crosse, le pontet, le quillon, le fût, le guidon, le tenon, la grenadière, la gachette. Or, jamais il ne dessine un revolver, ce qui ne lui serait pas difficile. Dans sa langue, revolver se dit ole kuga, soit petit fusil, de ole, petit et kuga, fusil. Il dessine alors deux fusils: un modèle standard et un petit fusil, ici le revolver. Le cadavre d'un chef est toujours représenté plus grand que le corps des deuilleurs et des ensevelisseurs, car l'expression na cawiri ba na kamo a: "cet homme dépasse le peuple», soit cet homme est un chef, doit être traduite exactement. Dans ses chants funèbres il parle d'un grand homme (notre expression est identique) et il prononce "gran-an-an-an-and», ce qui influence son burin.

Il va de soi que l'image des frères du chef, des personnes qui en dépendent, des vaincus et aujourd' hui des serviteurs, la clientèle en un mot, diffère en format de celle du chef: elle se compose de personnages plus petits que le personnage de référence, suivant le terme kamosari, de kamo, la personne et de sari, autre forme de petit. La nudité de ces hommes est tempérée par la présence d'un étui pénien en paille finement tressée, le bagayou. Cet attribut déterminatif ne pouvait manquer dans les représentations d'hommes. Un chef, un homme important, est décrit dans l'expression kamo ka vi na bu e, soit homme à phallus, la virilité étant traduite par $b u$. Les hommes importants apparaîtront donc en portant des étuis péniens considérables. Quoique fort respectés, les vieillards ne peuvent dissimuler certaines carences physiques. Ils doivent retenir leur bagayou en l'insérant dans leur ceinture: $n a$ cawiri o sua xie ro nemu dit-on à ce moment; le graveur tient compte de cette expression et le bagayou des anciens est relié à la ceinture par un 


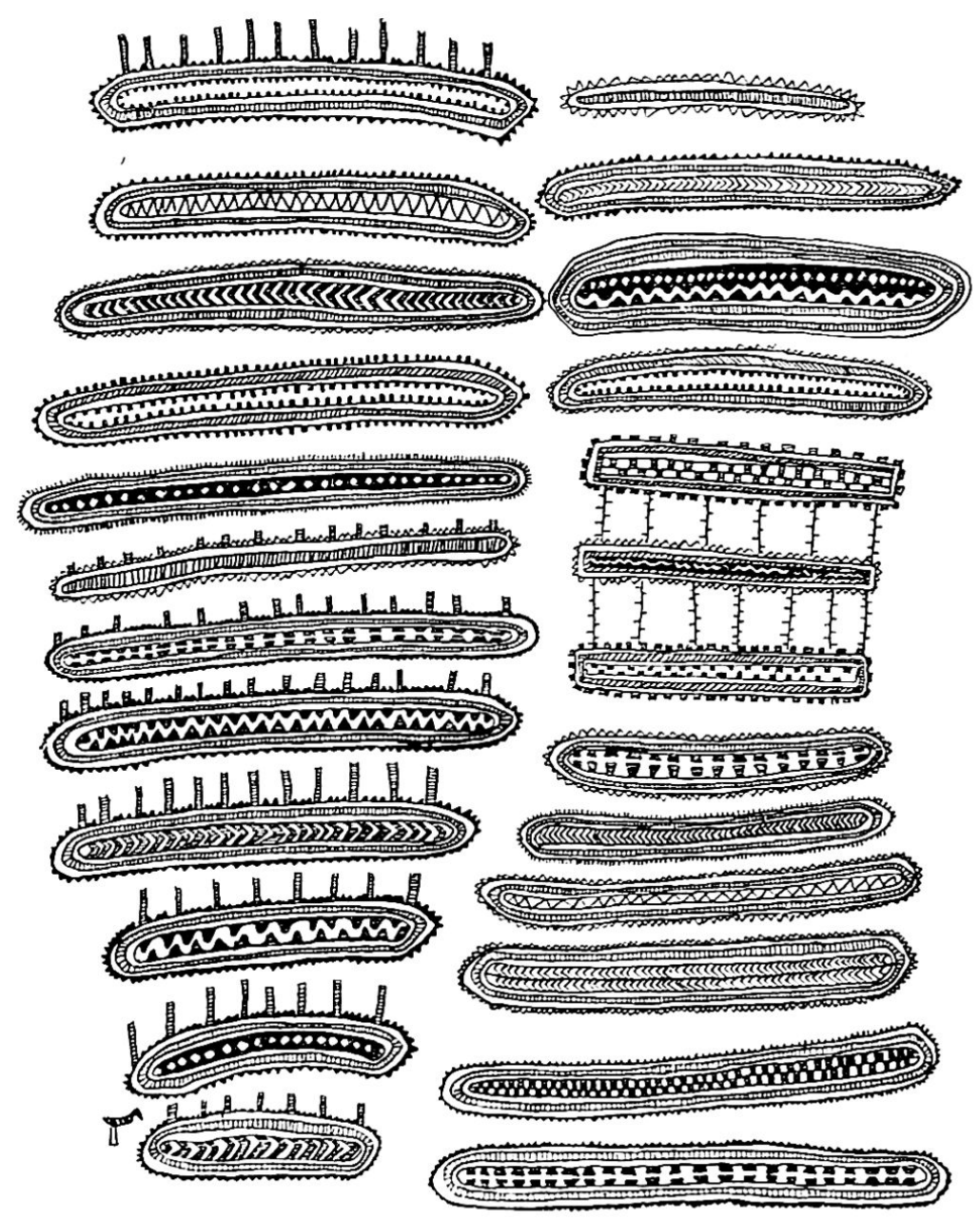

L'idéogramme représentant la plantation et la vie inventé par les Néo-Calédoniens

trait, qui représente l'insertion, car la perspective est absente de ces gravures où règne le procédé du rabattement. Toujours dans le chapitre viril si important pour ces hommes nus, on remarque que lors de fêtes villageoises, l'hôte est représenté avec un bagayou moins important que celui des invités ou de ses supérieurs, les oncles maternels: l'étiquette exige qu'il se fasse petit, c'est à dire qu'il soit modeste: cette réduction correspond à l'expression kamo ka pesari, l'homme qui se fait petit.

On sait que le défunt devient un bao, un dieu, un revenant, un fantôme. Il n'a alors que la tête et les membres, mais jamais de corps. Parlant d'un dieu endormi près de leur case, une jeune fille le décrit ainsi à sa sœur dans le conte des Deux filles de Weji: ... "Kamo re we na neme bari ae na seri para karo e...», ce qui, traduit littéralement, signifie: "homme là visage seul mais lui manquer tout le corps lui», soit, "cet homme n'est qu'un visage sans corps." C'est donc un dieu, un bao itinérant, comme nous en avons rencontré au cours de nos déchiffrements.

Une autre transcription littérale du langage par l'artiste canaque souligne l'association d'idée qui gouverne leur pensée. Le pêcheur enfile ses poissons par les ouïes sur un jonc. Cette brochette est nommée netu exoa, les poissons enfilés, $t u$ étant le passage d'une fibre par un orifice. Par analogie, le discours est traduit par une image phonétique semblable, notu, no étant la parole, et $t u$, comme dans l'expression précédente étant l'enfilage de la fibre. L'image graphique du discours sur ces bambous ou du message verbal est une série de losanges reliés par un trait fin: les paroles sont bien enfilées dans le graphique comme dans le phonétique. Une autre transcription, assez compliquée est celle du navigateur et riverain de la mer. Il se définit na uvara yenya na ke to nekuie, soit «le rester sur les vagues m'est coutumier». Un bambou d'une collection romaine montre cinq hommes figurés comme des Canaques de l'intérieur des terres, casse-tête en bec d'oiseau sur l'épaule, aigrette fichée dans les cheveux, debout sur une ligne vivrée horizontale surmontant des canots à balanciers et des bancs de poissons. Ici, ces canots, ces poissons, sont des déterminatifs insistant sur le côté maritime de la ligne vivrée, l'eau en mouvement perpétuel de la mer, la vague en un mot, et non quelque cours d'eau juste bon pour des simples terriens.

On peut citer l'usage assez subtil du suffixe $a$, qui est inversif ou privatif. Si manger se dit $\hat{a}$, jeûner se dit donc âa. Nous avons pu une fois, découvrir l'image d'une plantation abandonnée: les petits traits partant de haut en bas de la ligne de terre, contredisaient la direction habituelle de bas en haut, qui signifie germination. Ici, le préfix inversif $a$ a 
été traduit littéralement par la direction contraire à celle attribuée d'habitude au signe de fertilité. Un autre exemple apparait dans le port de la jupe des femmes qu'elle soit en fibre ou en tapa (écorce battue). Sur les bambous gravés elle figure sous l'aspect de deux petits traits horizontaux partant des hanches d'où descendent des petits trait verticaux. Dans les scènes érotiques évoquant et datant l'époque de la levée des tabous sexuels, sans intention graveleuse - le Canaque est peu salace et assez réservé sur ce sujet, nous dit $M$. Leenhardt - les traits verticaux vont de bas en haut. L'allusion est gracieusement subtile. Le graveur ne peut se permettre de présenter la femme sans jupe, car fréquemment elle apparait ainsi sur les bambous, dans les scènes de la vie courante, où seuls ses seins placés un peu n'importe où, servent de déterminatif du sexe. Le lecteur comprend l'allusion du graveur qui attend sa complicité; l'intelligence du procédé est visible.

Nous avons déjà mentionné la puissance d'abstraction des Néo-Calédoniens à la suite de M. Leenhardt et de J. Guiart, qui les étudièrent avec tant de profondeur. Nous savons que chez ces insulaires, le mot abstrait se forme par l'usage du mot $v i$, je vais. Une image fréquente sur les bambous montre un homme marchant ou en mouvement, avec quelque attribut: il ne s'agit pas ici d'un personnage en promenade, mais de la traduction d'un concept abstrait. Dans un cas très net apparaissant sur un bambou des collections du Musée d'Ethnographie de Bâle, on voit un homme porteur d'un tuteur; quand le Néo-Calédonien lisait ce dessin go vi na ka rha $a i$, je vais pour le planter tuteur, il comprenait immédiatement le sens exact soit: plantation $d u$ tuteur. Pour cette raison, qui ne nous est apparue clairement qu'en 1941, soit cinq ans après le début de nos tentatives de déchiffrement de cette mémoire collective que sont les bambous gravés, nous estimons que les innombrables petits personnages en mouvement repérés sur ces gravures traduisent des idées abstraites telles «je vais pour le pêcher poisson", soit la pêche en elle-même, et «je vais pour le tuer homme", soit le meurtre en tant que concept et non telle ou telle anecdote localisée ou personnalisée. Nous avons nommé cinématogrammes ces représentations animées destinées à transcrire le terme abstrait. Ici encore, le graphique est nettement subordonné au phonétique.

Il serait possible d'accumuler les exemples de cette soumission de l'image au mot, lui-même relié vigoureusement aux tours et détours de la pensée canaque. Ici, l'écriture, au sens très large du mot, suit avec précision l'expression parlée sans pour cela la travestir, la remplacer, la trahir. Elle n'est pas l'intrusion du matériel dans le spirituel, elle se contente d'être un truchement singulièrement capable de faire pénétrer l'ethnologue de 1966 dans le dé- dale d'une pensée disparue depuis plus d'un demisiècle, tout comme dans le détail d'une forme autochtone de culture fortement ébranlée par fes techniques et les pensers importés par la colonisation dès 1853 .

La surface de ces bambous est donc couverte de petits dessins représentant les activités humaines et matérielles de la vie des Canaques, chasse, pêche, navigation, agriculture, guerre auxquelles il faut ajouter l'image quasi photographique des cases dont les flèches faîtières caractéristiques permettent la localisation géographique. On note encore les cérémonies complexes, comme l'admirable Pilou d'un bambou du Musée d'Ethnographie de Genève ou de la préparation de ces fêtes, comme sur un bambou du Musée de l'Homme à Paris ou encore l'apparition du Dö̈bat, le grand dieu canaque, sur un exemplaire du Musée Pigorini, à Rome. Les allusions à la vie spirituelle inséparable de la vie quotidienne sont nombreuses. L'acculturation est manifeste dans les bambous gravés depuis un siècle. Ils forment un catalogue des étonnantes nouveautés importées par les Français, du cheval au chien, de la montre à la pioche, du voilier à l'attelage à bœufs, jusqu'à l'ivresse et la rixe entre Blancs. En effet, seuls sans doute de tous les peuples de la Terre, les Néo-Calédoniens n'avaient jamais inventé même une boisson fermentée, encore moins l'alcool: l'eau suffisait à les désaltérer. De là, les observations manquant d'indulgence sur la tenue de marins au béret à rubans ou de colons attablés devant des étalages de boứrilles et de verres. Tout ce matériel gravé est précieux et il arrive fréquemment qu'une vieille photo, qu'une vieille carte postale confirment la précision du message canaque. A ce moment, les incomparables documents publiés par Fritz Sarasin, de Bâle, sont d'un secours inappréciable.

Lorsque les frappeurs du Soleil mentionnés antérieurement ont blessé ce Soleil pour faire tomber la pluie, ils vont déposer près d'une pièce d'eau un paquet magique, le bore, et l'offrent aux ancêtres pour les inciter à faire pleuvoir. Ce bore contient divers objets associés à la pluie selon les catégories locales, des cordelettes de coco et de poil de roussette, des morceaux de roseau et de filet de pêche, des cailloux-image de l'igname, des feuilles de plantes humides et de plantes sèches, le tout étant enveloppé dans un morceau d'écorce bien ficelé. Ce paquet contient donc tous les éléments capables d'influencer la croissance de l'igname, nourriture de base de l'île. Ce dépôt a inspiré aux Néo-Calédoniens un dépassement de la pictographie élémentaire, pourtant si précieuse pour l'information ethnographique. Ils ont franchi le stade de la simple traduction imagée de l'expression parlée. Ils on matérialisé ici le mental sans passer par le signe phonétique: ils ont traduit à leur manière 
une formule mentale pleine de sous-entendus mystiques: ils ont donc créé un idéogramme subtil et compréhensible.

Ils ne pouvaient figurer le bore sans inscrire tous les éléments de cet objet culturel, ce qui eût été motif à confusion et à explications graphiques compliquées. Voulant signifier le concept de fertilité du sol, de nourriture essentielle, de garantie de vie pour le groupe social, et peut-être aussi, d'année agricole, plus courte que l'année sidérale, ils ont dessiné un cartouche ovale, correspondant à l'enveloppe du bore. A l'intérieur, ils ont accumulé tous les éléments de la culture de l'igname, cette culture protégée par les cérémonies d'invocation à la pluie, qui ont lieu en janvier, alors que le débroussage avait été effectué en juillet et que le défonçage du sol et la plantation des ignames avaient eu lieu en octobre. Ces moments culturaux apparaissent sous la forme de lignes de triangles (la terre labourée), les traits verticaux (les tuteurs), les lignes vivrées (l'eau), les petits rectangles sous les lignes de terre (les ignames-plantons et les ignames à récolter), les traits horizontaux sortant des tuteurs (la plante arrivée à maturité). Tout le calendrier agricole est schématisé dans ces cartouches qui ne sont pas que de simples motifs décoratifs, mais bien la traduction d'une ardente prière graphique enveloppée dans le cartouche protecteur tout comme les objets d'intercession sont serrés dans l'enveloppe d'écorce, tout comme aussi les litanies des invocations jaillissent de l'âme canaque lors des cérémonies collectives. Ils ont donc figuré au troisième degré le résultat des opérations magiques qui vont de l'appel aux vents alizés, de juillet, jusqu'au rite de la pluie de janvier, avec le dépôt des bouquets sacrés et des pierres magiques. Pour une fois, le passage du spirituel au graphique s'exprime sans l'intermédiaire du phonétique, le mot bore (paquet magique) étant transformé en un symbole non assimilable immédiatement quant à sa forme et à son contenu matériel. Cette synthèse étonnante est l'œuvre de paysans qui longtemps passèrent pour de sombres brutes, jusqu'à la publication par Maurice Leenhardt dès 1930 , du résultat de ses enquêtes magistrales de plus d'un quart de siècle. La présence étrangère et le poids des importations mentales ont arrêté à jamais cette marche autonome et originale vers une idéographie intellectualisée.
Suivre le cheminement mental de ces cultivateurs d'ignames jusqu'à la création d'un idéogramme aussi subtil et abstrait que celui qui vient d'être décrit est une expérience rare. Dans ce genre de recherches, il est peu fréquent d'assister à l'évolution de signes simples, de plus en plus schématisés, toujours chargés de sens affectif, jusqu'à leur transformation en symboles dont l'association, pleine de révérence, est l'égide d'une société isolée, respectueuse des rites instaurés par le geste des ancêtres et capable par elle même d'inventer des façons culturales propres à assurer l'existence des tribus, pour autant que lézards et bao bénissent les travaux de leurs pieux zélateurs. Le bore et son idéogramme traduisent la piété et la confiance des planteurs néocalédoniens, inventeurs de cette gravure sur bambou devenue une source de connaissance pour l'ethnographie.

\section{Zusammenfassung}

Eine Reihe völkerkundlicher Museen besitzt mit feinen Zeichnungen bedeckte Bambusstäbe aus Neu-Kaledonien. Die Darstellungen geben halbrealistische Szenen aus dem materiellen Leben des dort lebenden, geographisch wie geistig isolierten Volkes wieder; ähnlich wie schematischen Figuren ist ihnen ein tiefer Symbolgehalt zu eigen. Die Deutung setzt freilich viel Erfahrung voraus; auch verlangt sie gute Kenntnis der lokal-ethnographischen Verhältnisse wie des Seelenzustandes dieser Kannibalen, deren brutal wirkendes Äußeres lange Zeit hindurch nicht auf die abstrakten Formen ihres Gedankengutes und auf ihre autonome Kraft der Selbstbesinnung schließen ließ. Heute verstehen die gleichen Kanaken diese in die Völkerkundemuseen geretteten Archive nicht mehr zu lesen.

Die Bambus-Einritzungen sind nicht Ergebnis einer Spielerei. Sie bilden ganz im Gegenteil die schriftliche UUbertragung des gesprochenen Wortes, das seinerseits der intellektuellen Verfassung der Eingeborenen Rechnung trägt, das heißt deren ständigem Verwechseln von Wirklichkeit, Mythos und Symbol Ausdruck verleiht. Die Zeichnungen, die vom scharf gestochenen kleinen Bild zum geometrischen Motiv voller Mystik den Bogen spannen, sind denn in überraschender und seltener Treue Spiegel des einmal mündlich Geäußerten. 